\section{Frecuencia de displasia epitelial y carcinoma escamoso en mucosa oral y orofaríngea en Chile, entre los años 1990 y 2009}

\author{
CAROLINA MARTÍNEZ $Z^{1, a}$, MARCELA HERNÁNDEZ $Z^{1, a, b}$, \\ BENJAMÍN MARTÍNEZ ${ }^{2, a}$, DANIELA ADORNO ${ }^{1, a}$
}

\section{Frequency of oral squamous cell carcinoma and oral epithelial dysplasia in oral and oropharyngeal mucosa in Chile}

Background: Oral cancer in Chile corresponds approximately to $1.6 \%$ of all cancer cases. There are few studies about oral epithelial dysplasia and oral squamous cell carcinoma in the Chilean population. Aim: To determine the frequency of hyperkeratosis, mild, moderate and severe oral epithelial dysplasia, in situ carcinoma and squamous cell carcinoma of the oral and oropharyngeal mucosa in a registry of the Oral Pathology Reference Institute of the Faculty of Dentistry, Universidad de Chile, in a ten years period. Material and Methods: Review of clinical records and pathological plates of 389 patients, obtained between 1990 and 2009. Cases were selected according to their pathological diagnosis, including hyperkeratosis, oral epithelial dysplasia, in situ carcinoma, squamous cell carcinoma and verrucous carcinoma. Results: Forty four percent of cases were squamous cell carcinoma, followed by hyperkeratosis in $37 \%$ and mild epithelial dysplasia in 11\%. Squamous cell carcinoma was more common in men aged over 50 years. Most of the potentially malignant disorders presented clinically as leukoplakia and squamous cell carcinoma were clinically recognized as cancer. Conclusions: In this study, men aged over 50 years are the highest risk group for oral cancer. Early diagnosis is deficient since most of these lesions were diagnosed when squamous cell carcinoma became invasive. Leukoplakia diagnosis is mostly associated with hyperkeratosis and epithelial dysplasia, therefore biopsy of these lesions is mandatory to improve early diagnosis.

(Rev Med Chile 2016; 144: 169-174)

Key words: Epidemiology; Leukoplakia; Mouth neoplasms; Neoplasms, squamos cell.

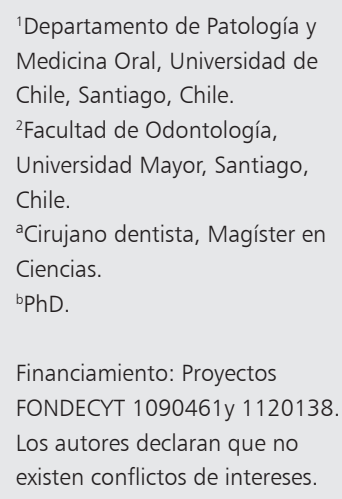

Recibido el 13 de marzo de 2015 aceptado el 5 de noviembre de 2015.

\section{Correspondencia a:}

Dra. Daniela Adorno Farías

Teléfono: 29781808

daniadorno@gmail.com
$\mathrm{E}$ 1 cáncer oral es una enfermedad frecuente en muchos países y en Chile se estima que corresponde a 1,6\% del total de cánceres. El carcinoma de células escamosas (CE) es el tipo más frecuente de cáncer oral ${ }^{1,2}$. Previo a la etapa del desarrollo del CE se agrupan los desórdenes potencialmente malignos (DPM) que se refie- ren a determinadas alteraciones morfológicas, dentro de las cuales, algunas pueden tener un potencial aumentado de sufrir transformación maligna. Además, los DPM pueden indicar un mayor riesgo de desarrollar cáncer en cualquier lugar de la mucosa oral aparentemente normal y no solamente en un sitio específico afectado. Se 
prefiere el término potencialmente maligno al precancencerizable o premaligno, pues no todas estas alteraciones progresarán necesariamente a cáncer ${ }^{3-5}$.

La tasa de transformación maligna de los DPM a carcinoma escamoso varía principalmente según la población, sus hábitos y la localización de la lesión ${ }^{5,6}$. El tabaco, en sus variantes fumable o masticable, es un reconocido carcinógeno. El riesgo más elevado afecta a los fumadores pesados y sin filtro, especialmente después de muchos años de uso. El alcohol puede actuar como promotor, teniendo un efecto sinérgico con el tabaco ${ }^{7-9}$. Lesiones en piso de boca, cara ventral y lateral de lengua tienen mayor riesgo de sufrir transformación maligna ${ }^{3,5,10-12}$. A mayor edad aumenta la probabilidad de sufrir lesiones potencialmente malignas y cáncer, con un incremento de cáncer oral a los 60 años, probablemente debido a que las mutaciones que conducen a la transformación maligna se van sumando ${ }^{9}$. Algunos autores también relacionan el virus papiloma humano con la transformación maligna, especialmente los subtipos 16 y 18 ; sin embargo, esta relación es controversial, pues tales virus también se pueden encontrar en el epitelio de la mucosa normal ${ }^{13,14}$.

El CE generalmente está precedido por una variedad de alteraciones celulares y tisulares compatibles con carcinoma, que están restringidas al epitelio y que se denomina displasia epitelial $(\mathrm{DE})^{3}$. Evidencias señalan que mientras más severa es la DE, mayor probabilidad de transformación maligna, por lo que se destaca la importancia de una adecuada caracterización de los rasgos histopatológicos de cada subtipo de $\mathrm{DE}^{9,15}$. El sistema de clasificación más ampliamente utilizado es el ratificado por la OMS en 2005, el cual distingue entre DE leve, moderada, severa y carcinoma in situ (CIS). Las características de cada entidad se agrupan en dos categorías: cambios en la arquitectura epitelial y atipias celulares ${ }^{5,16}$.

Conociendo que en la literatura actual hay escasos estudios sobre la frecuencia de DE y CE en mucosa oral realizados en la población chilena, el objetivo de este estudio es determinar la frecuencia de hiperqueratosis, DE leve, moderada y severa, CIS y CE en biopsias de mucosa oral y orofaríngea pertenecientes al Instituto de Referencia de Patología Oral (IREPO) de la Universidad de Chile, desde 1990 hasta 2009.

\section{Materiales y Método}

Se llevó a cabo un estudio descriptivo retrospectivo en el cual se realizó una revisión del registro de fichas y de placas histológicas registradas en el Instituto de Referencia de Patología Oral (IREPO) de la Facultad de Odontología, Universidad de Chile, entre los años 1990 y 2009.

Se seleccionaron las fichas de pacientes con biopsia proveniente de mucosa oral u orofaríngea y diagnóstico histopatológico correspondiente a hiperqueratosis, DE leve, moderada y severa, CIS, CE y carcinoma verrucoso; y con diagnóstico clínico correspondiente a leucoplasia, eritroplasia, cáncer u otro. Fueron excluidas del estudio aquellos casos en que no se pudo establecer o confirmar el diagnóstico histopatológico, y aquellos que carecían de información respecto a la ubicación de la lesión.

Se consignaron los siguientes datos generales y clínicos de los casos incluidos: género, edad, ubicación, diagnóstico clínico y diagnóstico histopatológico. Luego se revisaron las placas histológicas, directamente en un microscopio óptico (Olympus, BX4, Tokio, Japón), para confirmar el diagnóstico histopatológico. Se excluyeron aquellas placas cuya muestra no fuera representativa de la lesión $o$ ésta se encontrase mal procesada.

La confirmación del diagnóstico de DE y CIS se hizo de acuerdo a los criterios de la OMS:

- DE leve: Mínimas atipias celulares, limitadas al estrato basal o parabasal del epitelio.

- DE moderada: Alteraciones del epitelio hasta la mitad del estrato espinoso. Atipias tales como hipercromatismo, pleomorfismo, mitosis aumentadas y anormales en el estrato basal. Cambios en la arquitectura epitelial como pérdida de la polaridad, hiperplasia y crestas epiteliales en forma de gotas.

- DE severa: Cambios muy prominentes desde el estrato basal hasta sobrepasar el tercio superior del epitelio. Se observa marcado pleomorfismo, núcleo anormalmente grande con múltiples nucléolos, mitosis suprabasales y anormales, pérdida de estratificación, disqueratosis, perlas de queratina, crestas epiteliales con marcada forma de gotas.

- CIS: Los cambios se observan en todo el grosor del epitelio.

Este estudio retrospectivo fue aprobado por el 
Comité de Ética de la Facultad de Odontología de la Universidad de Chile y se llevó a cabo de acuerdo con las Normas Consolidadas de Declaración Reporting Trials, CIOMS 2008 y la Declaración de Helsinki de 1975 revisada en 2000. Cualquier detalle que pudiera revelar la identidad de los sujetos estudiados fueron omitidos.

El análisis histológico fue realizado por un sólo observador. Los datos se procesaron en el software Systat 13.

\section{Resultados}

Se incluyeron 389 pacientes que cumplían con criterios de inclusión y exclusión; de estos, ocho pacientes tenían dos biopsias de distintas ubicaciones realizadas el mismo año y dos pacientes tenían dos biopsias en distintas ubicaciones y realizadas en años distintos. Un total de $147(36,8 \%)$ casos correspondieron a hiperqueratosis, $42(10,5 \%)$ a DE leve, $27(6,8 \%)$ a DE moderada, $4(1 \%)$ a carcinoma verrucoso y $3(0,8 \%)$ a DE severa, mientras que 176 casos $(44,1 \%)$ correspondieron a CE (Figura 1).

De los ocho pacientes con biopsias en distintas ubicaciones, cinco presentaron diagnósticos histopatológicos que variaron según la ubicación y de los dos pacientes con biopsias realizadas en distintos años y en distintas ubicaciones, ambos presentaron DE en la primera y CE en la segunda.

Según rango de edad, todas estas lesiones fueron más frecuentes después de los 50 años, destacándose principalmente los casos de CE, en que $149(86,13 \%)$ se presentaron a partir de los 50 años y sólo $24(13,87 \%)$ antes de esta edad (Tabla 1). El promedio de edad para CE fue de 62,99 años (DS 14,46), para hombres fue 62,8 años (DS 12,8 ) y para mujeres 63,25 años (DS 16,64) (datos no mostrados).

En relación con la distribución por género, el CE fue más frecuente en hombres (101; 58,4\%), mientras que la DE fue más frecuente en mujeres $(45 ; 65,2 \%)$ y las hiperqueratosis presentaron frecuencia similar en ambos géneros (Tabla 1).

En términos generales, las ubicaciones más comunes para CE correspondieron a reborde o encía $(71 ; 29,7 \%)$, lengua $(50 ; 20,9 \%)$ y piso de boca $(43 ; 18,0 \%)$; las DE fueron más frecuentes en lengua $(26 ; 31,3 \%)$ y reborde o encía $(6 ; 7,2 \%)$, y las hiperqueratosis en reborde o encía $(52 ; 34,2 \%)$ y mucosa bucal $(42 ; 27,6)$ (Tabla 1$)$.

La mayoría de los CE $(138 ; 78,41 \%)$ fueron reconocidos clínicamente como cáncer y la mayoría de las DE $(40 ; 55,56 \%)$ e hiperqueratosis $(107 ; 72,79 \%)$ se presentaron como leucoplasia (Tabla 1).

Dentro de las lesiones correspondientes a DE es importante destacar que una fue diagnosticada clínicamente con un diagnóstico histopatológico (DE/carcinoma) y otras con diagnósticos clínicos que no correspondían a DPM (una como penfigoide de membranas mucosas, dos fibromas irritativos, dos queratosis, cuatro úlceras y siete papilomas) (Figura 2).

Finalmente, dentro de los CE también se encontraron diagnósticos clínicos de lesiones que no correspondían con la presentación habitual de cáncer, tales como un épulis fisurado, un fibroma verdadero, dos fibromas irritativos, dos granulomas piogénicos, una hiperplasia gingival, una lesión eritematosa, una lesión fibroósea, una lesión granulomatosa inespecífica, dos osteomielitis, un papiloma, una tuberculosis y una úlcera de tipo herpético.

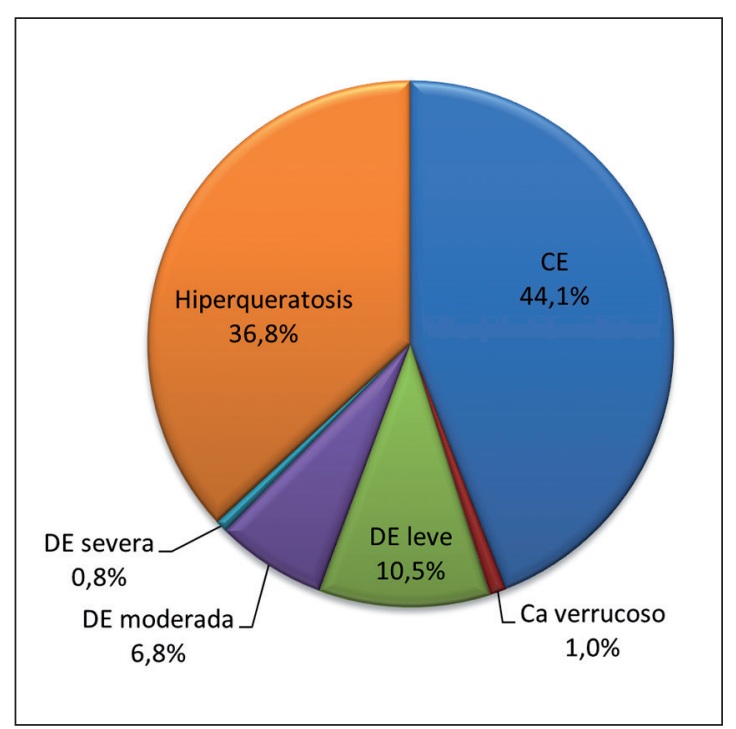

Figura 1. Frecuencia de hiperqueratosis, displasia epitelial (DE), carcinoma verrucoso y carcinoma escamoso (CE) en mucosa oral y orofaríngea según diagnóstico histopatológico $(n=389)$. 
Tabla 1. Datos epidemiológicos de los pacientes

\begin{tabular}{|c|c|c|c|c|c|c|c|c|}
\hline \multirow[b]{2}{*}{ Variables } & \multicolumn{2}{|c|}{$\begin{array}{l}\text { Carcinoma } \\
\text { escamoso }\end{array}$} & \multicolumn{2}{|c|}{$\begin{array}{l}\text { Carcinoma } \\
\text { verrucoso }\end{array}$} & \multicolumn{2}{|c|}{$\begin{array}{l}\text { Displasia } \\
\text { epitelial }\end{array}$} & \multicolumn{2}{|c|}{ Hiperqueratosis } \\
\hline & $\mathbf{n}$ & $\%$ & $\mathbf{n}$ & $\%$ & $\mathbf{n}$ & $\%$ & $\mathbf{n}$ & $\%$ \\
\hline \multicolumn{9}{|l|}{ Edad } \\
\hline Sin dato & 0 & & 0 & & 0 & & 1 & 0,70 \\
\hline $15-49$ & 24 & 13,87 & 0 & & 21 & 30,43 & 60 & 41,96 \\
\hline$>50$ & 149 & 86,13 & 4 & 100 & 48 & 69,57 & 82 & 57,34 \\
\hline \multicolumn{9}{|l|}{ Género } \\
\hline Sin dato & 1 & 0,58 & 0 & & 0 & & 0 & \\
\hline Femenino & 71 & 41 & 2 & 50 & 45 & 65,22 & 72 & 50,35 \\
\hline Masculino & 101 & 58,38 & 2 & 50 & 24 & 34,78 & 71 & 49,65 \\
\hline \multicolumn{9}{|l|}{ Ubicación } \\
\hline Lengua & 50 & 20,92 & 3 & 75 & 26 & 31,33 & 25 & 16,45 \\
\hline Piso boca & 43 & 17,99 & 0 & & 6 & 7,23 & 6 & 3,95 \\
\hline Mucosa bucal & 31 & 12,97 & 0 & & 13 & 15,66 & 42 & 27,63 \\
\hline Mucosa labial & 1 & 0,42 & 0 & & 0 & & 1 & 0,66 \\
\hline Reborde y encía & 71 & 29,71 & 0 & & 21 & 25,30 & 52 & 34,21 \\
\hline Paladar duro & 15 & 6,28 & 1 & 25 & 12 & 14,46 & 7 & 4,61 \\
\hline Trígono retromolar & 4 & 1,67 & 0 & & 1 & 1,20 & 15 & 9,87 \\
\hline Orofaringe & 21 & 8,79 & 0 & & 4 & 4,82 & 4 & 2,63 \\
\hline \multicolumn{9}{|l|}{ Dx clínico } \\
\hline Sin dato & 4 & 2,27 & 0 & & 0 & & 0 & \\
\hline Cáncer & 138 & 78,4 & 0 & & 6 & 8,33 & 1 & 0,68 \\
\hline Eritroplasia & 1 & 0,57 & 0 & & 1 & 1,39 & 1 & 0,68 \\
\hline Leucoplasia & 0 & & 2 & 50 & 40 & 55,56 & 107 & 72,79 \\
\hline Otro & 33 & 18,75 & 2 & 50 & 25 & 34,72 & 38 & 25,85 \\
\hline
\end{tabular}

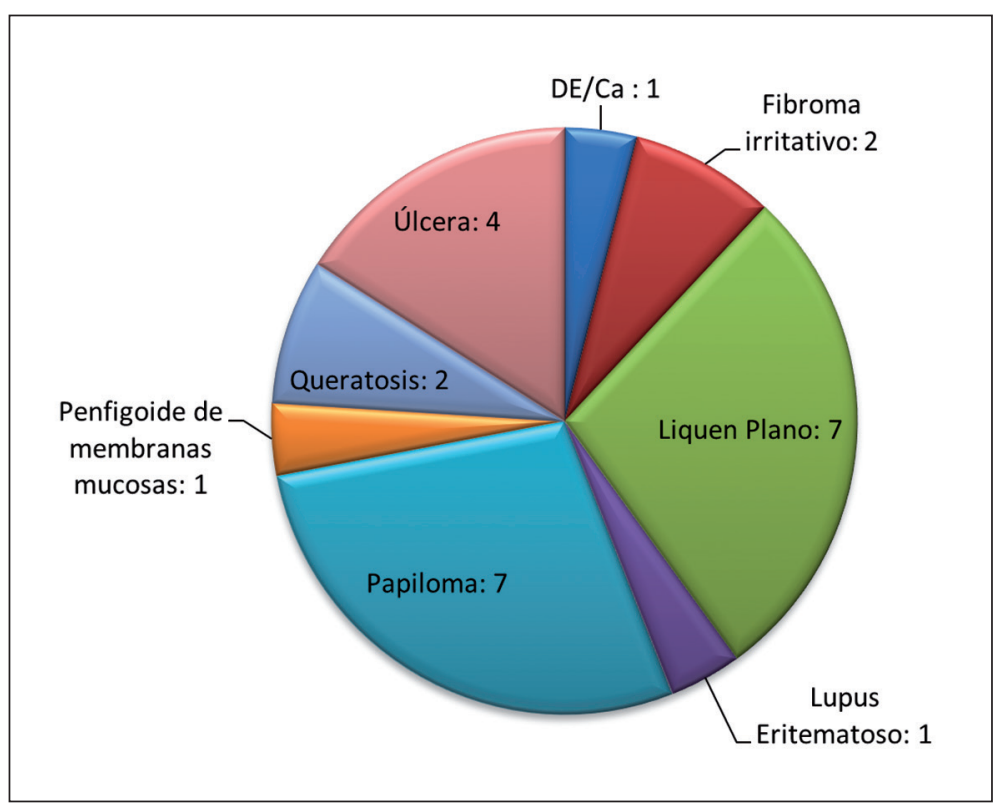

Figura 2. Otros diagnósticos clínicos de displasia epitelial $(n=21)$. DE/Ca: Diagnóstico clínico de displasia epitelial o carcinoma. 


\section{Discusión}

El diagnóstico clínico e histopatológico de los DPM puede ser difícil en algunos casos debido a la subjetividad y falta de una evaluación sistemáti$\mathrm{ca}^{17,18}$. Sin embargo, su identificación oportuna es de vital importancia, ya que actualmente se considera que incluso la mucosa aparentemente normal en un paciente que presenta un DPM puede albergar cambios sugerentes de transformación maligna, por lo que el cáncer podría originarse en un sitio distinto al de la lesión ${ }^{6}$. Acorde con esto, encontramos diagnósticos histopatológicos diferentes según la ubicación de la lesiónen cinco pacientes. Además, en dos pacientes se diagnosticó primeramente DE y posteriormente, en otra ubicación, CE.

Generalmente, hiperqueratosis, DE y CE son lesiones que se presentan en pacientes mayores. En nuestro estudio observamos que todas estas lesiones se encuentran mayormente a partir de los 50 años, lo cual está en concordancia con otros estudios ${ }^{9,18}$. Sin embargo, existe un porcentaje importante de displasias epiteliales y carcinomas escamosos orales que ocurren en individuos más jóvenes, que podrían atribuirse a factores de riesgo no clásicos, tales como infección por virus papiloma humano de alto riesgo, factores genéticos e inmunodeficiencia ${ }^{14}$.

En este estudio se encontró mayor frecuencia de CE en hombres, lo cual es similar a otros reportes $^{9}$, pero, por otro lado, los casos de DE fueron casi el doble más en mujeres que en hombres; esto podría deberse a que las mujeres consultan con mayor antelación que los hombres.

La ubicación más frecuente de DE fue lengua, seguido de reborde y encía. Otros estudios también han encontrado mayor frecuencia en estas ubicaciones ${ }^{18}$. En los casos de CE, las localizaciones más frecuentes fueron reborde y encía, lengua y piso de boca, en concordancia con un estudio previo realizado en Temuco, Chile ${ }^{2}$. Estudios previos han identificado a la lengua, especialmente las caras lateral y ventral, como la ubicación más frecuente de carcinoma oral, seguido de piso de boca. Estas áreas podrían estar en mayor riesgo por dos razones: primero, porque los agentes carcinógenos se mezclan con la saliva, acumulándose en esta zona y bañándola constantemente. Segundo, porque el epitelio de estos sitios es más delgado y no queratinizado, lo cual brinda menor protección contra agentes carcinógenos ${ }^{9}$.
En relación a displasia encontramos mayor frecuencia de DE leve, seguida de DE moderada y DE severa. Esto es similar a lo encontrado en reportes previos ${ }^{18}$. La presencia de DE sigue considerándose el predictor más fuerte de una futura transformación maligna ${ }^{6}$; sin embargo, cabe considerar que lesiones no displásicas también pueden devenir en $\mathrm{CE}^{5,6,15}$.

Según su presentación clínica, la mayoría de los CE se diagnosticaron como cáncer (138, 78,41\%). Además, 4 casos $(2,73 \%)$ no presentaban diagnóstico clínico y $33(18,8 \%)$ presentaban otros diagnósticos, dentro de los cuales se encontraron lesiones que no tienen ninguna relación con $\mathrm{CE}$ o DPM, pero ninguno fue diagnosticado como leucoplasia y sólo uno como eritroplasia. Estos resultados contrastan con lo encontrado por otros estudios $^{19,20}$ en los cuales algunas leucoplasias resultaron ser CE.

Nuestros resultados llaman la atención y sugieren que el diagnóstico de CE se realiza cuando éste presenta signos clínicos característicos de una etapa avanzada, tales como ulceración, bordes indurados, sangrado y sobrecrecimiento tumo$\mathrm{ral}^{20}$. A pesar de la accesibilidad de la cavidad oral al examen clínico directo, nuestros resultados demuestran que el CE oral no se detecta hasta una fase tardía9. Además, llama la atención que se confunda con lesiones tales como hiperplasia gingival o fibroma irritativo, entre otras, enfatizando la necesidad de alertar a la comunidad odontológica sobre las posibles manifestaciones clínicas de CE y DPM, a fin de que éste se pueda pesquisar precozmente y de manera adecuada.

En cuanto a DE, también encontramos diagnósticos clínicos que no corresponden a DPM. En relación a esto se propone excluir otras condiciones, desórdenes o enfermedades mediante una cuidadosa historia y examen clínico, acercando así el diagnóstico clínico inicial de las lesiones observadas a sus consecuentes diagnósticos histopatológicos ${ }^{6}$.

La variedad de diagnósticos clínicos iniciales encontrados en el estudio en los casos asociados a DE y a CE se da una vez que los casos revisados provienen de distintos odontólogos tratantes.Sin embargo, esto no es una limitante para el trabajo, y a su vez, muestra la necesidad de toma de conciencia por parte de los clínicos generales que deben mejorar sus conocimientos en el área de patología oral y que deben derivar sus pacientes 
a especialistas siempre que no tenga claro el diagnóstico inicial. Un adecuado diagnóstico clínico determina un plan de tratamiento óptimo y una toma de biopsia incisional o escisional pertinente para cada caso.

El presente es el primer estudio que determinar la frecuencia de DE leve, moderada y severa, CIS y CE de mucosa oral y orofaríngea en la población chilena. Podemos concluir que la mayoría de los casos correspondieron a CE y los hombres mayores de 50 años al principal grupo de riesgo. El diagnóstico temprano es deficiente, ya que la mayor parte son diagnosticados como cáncer propiamente tal, es decir, cuando éste es bastante evidente y presenta sus características clásicas. El diagnóstico de leucoplasia está mayormente asociado con hiperqueratosis y DE, por lo tanto, la biopsia de estas lesiones es mandatoria para mejorar de forma significativa el diagnóstico temprano.

\section{Referencias}

1. Riera P, Martínez B. Morbilidad y mortalidad por cáncer oral y faríngeo en Chile. Rev Med Chile 2005; 133 (5): 555-63.

2. Guzmán P, Villaseca M, Antonio L, Araya J, Aravena P, Cravero C, et al. Carcinoma epidermoide oral y orofaríngeo. Estudio clínico-patológico. Rev Chil Cir 2011; 63 (3): 250-6.

3. Dost F, Le Cao K, Ford PJ, Ades C, Farah CS. Malignant transformation of oral epithelial dysplasia: a real-world evaluation of histopathologic grading. Oral Surg Oral Med Oral Pathol Oral Radiol 2014; 117 (3): 343-52.

4. van der Waal I. Potentially malignant disorders of the oral and oropharyngeal mucosa; present concepts of management. Oral Oncol 2010; 46 (6): 423-5.

5. Warnakulasuriya S, Reibel J, Bouquot J, Dabelsteen E. Oral epithelial dysplasia classification systems: predictive value, utility, weaknesses and scope for improvement. J Oral Pathol Med 2008; 37 (3): 127-33.

6. Warnakulasuriya S, Johnson NW, van der Waal I. Nomenclature and classification of potentially malignant disorders of the oral mucosa. J Oral Pathol Med 2007; 36 (10): 575-80.

7. Jaber MA, Porter SR, Gilthorpe MS, Bedi R, Scully C. Risk factors for oral epithelial dysplasia--the role of smoking and alcohol. Oral Oncol 1999; 35 (2): 151-6.

8. Jaber MA, Porter SR, Scully C, Gilthorpe MS, Bedi R. The role of alcohol in non-smokers and tobacco in non- drinkers in the aetiology of oral epithelial dysplasia. Int J Cancer 1998; 77 (3): 333-6.

9. Neville BW, Day TA. Oral cancer and precancerous lesions. CA Cancer J Clin 2002; 52 (4): 195-215.

10. Islam MN, Kornberg L, Veenker E, Cohen DM, Bhattacharyya I. Anatomic site based ploidy analysis of oral premalignant lesions. Head Neck Pathol 2010; 4 (1): $10-4$.

11. Napier SS, Speight PM. Natural history of potentially malignant oral lesions and conditions: an overview of the literature. J Oral Pathol Med 2008; 37 (1): 1-10.

12. Reibel J. Prognosis of oral pre-malignant lesions: significance of clinical, histopathological, and molecular biological characteristics. Crit Rev Oral Biol Med 2003; 14 (1): 47-62.

13. Miller CS, White DK. Human papillomavirus expression in oral mucosa, premalignant conditions, and squamous cell carcinoma: a retrospective review of the literature. Oral Surg Oral Med Oral Pathol Oral Radiol Endod 1996; 82 (1): 57-68.

14. Majchrzak E, Szybiak B, Wegner A,Pienkowski J, Pazdrowski J, Luczewski, et al. Oral cavity and oropharyngeal squamous cell carcinoma in young adults: a review of the literature. Radiol Oncol 2014; 48 (1): 1-10.

15. Sperandio M, Brown AL, Lock C, Morgan PR, Coupland $\mathrm{VH}$, Madden PB, et al. Predictive value of dysplasia grading and DNA ploidy in malignant transformation of oral potentially malignant disorders. Cancer Prev Res (Phila) 201; 6 (8): 822-31.

16. Fleskens S, Slootweg P. Grading systems in head and neck dysplasia: their prognostic value, weaknesses and utility. Head Neck Oncol 2009; 1: 11.

17. Kujan O, Khattab A, Oliver RJ, Roberts SA, Thakker N, Sloan P. Why oral histopathology suffers inter-observer variability on grading oral epithelial dysplasia: an attempt to understand the sources of variation. Oral Oncol 2007; 43 (3): 224-31.

18. Pereira Jdos S, Carvalho Mde V, Henriques AC, de Queiroz Camara TH, Miguel MC, Freitas Rde A. Epidemiology and correlation of the clinicopathological features in oral epithelial dysplasia: analysis of 173 cases. Ann Diagn Pathol 2011; 15 (2): 98-102.

19. Hosni ES, Salum FG, Cherubini K, Yurgel LS, Figueiredo MA. Oral erythroplakia and speckled leukoplakia: retrospective analysis of 13 cases. Braz J Otorhinolaryngol 2009; 75 (2): 295-9.

20. Lee JJ, Hung HC, Cheng SJ, Chen YJ, Chiang CP, Liu BY, et al. Carcinoma and dysplasia in oral leukoplakias in Taiwan: prevalence and risk factors. Oral Surg Oral Med Oral Pathol Oral Radiol Endod 2006; 101 (4): 47280. 